\title{
Magnetic Heating Stimulated Cargo Release with Dose Control using Multifunctional MR and Thermosensitive Liposome
}

\author{
Sayoni Ray ${ }^{1}$, Chi-An Cheng ${ }^{2 *}$, Wei Chen ${ }^{*}$, Zhao Li1 ${ }^{*}$, Jeffrey I. Zink ${ }^{\circledR}$, Yung-Ya Lin $^{1 凶}$ \\ 1. Department of Chemistry and Biochemistry, University of California, Los Angeles, CA 90095, USA \\ 2. Department of Bioengineering, University of California, Los Angeles, CA 90095, USA \\ ${ }^{*}$ Co-second authors; these authors contributed equally to this work. \\ $\bowtie$ Corresponding authors: zink@chem.ucla.edu (Jeffrey I. Zink), yylin@chem.ucla.edu (Yung-Ya Lin) \\ (c) Ivyspring International Publisher. This is an open access article distributed under the terms of the Creative Commons Attribution (CC BY-NC) license \\ (https://creativecommons.org/licenses/by-nc/4.0/). See http://ivyspring.com/terms for full terms and conditions.
}

Received: 2018.12.06; Accepted: 2019.04.04; Published: 2019.04.19

\begin{abstract}
Rationale: Magnetic resonance imaging (MRI) is one of the most widely used diagnostic tools in the clinic. In this setting, real-time monitoring of therapy and tumor site would give the clinicians a handle to observe therapeutic response and to quantify drug amount to optimize the treatment. In this work, we developed a liposome-based cargo (cancer drugs) delivery strategy that could simultaneously monitor the real-time alternating magnetic field-induced cargo release from the change in MRI relaxation parameter $R_{1}$ and the location and condition of liposome from the change in $R_{2}$. The tumor site can then be monitored during the cargo release because liposomes would passively target the tumor site through the enhanced permeability and retention (EPR) effect. Physical insights from the experimental results and corresponding Monte Carlo spin dynamics simulations were also discussed.

Methods: Superparamagnetic iron oxide (SPIO) nanoparticles, diethylenetriaminepentaacetic acid gadolinium(III) (Gd(III)-DTPA), and a model cancer drug (fluorescein) were co-loaded in PEGylated thermosensitive liposomes. The liposomes were characterized by transmission electron cryo-microscopy (cryoTEM), dynamic light scattering (DLS), and inductively coupled plasma optical emission spectrometry (ICP-OES). Alternating magnetic field (AMF) was used to create controlled mild hyperthermia $\left(39-42^{\circ} \mathrm{C}\right)$ and facilitate controlled cargo (fluorescein) release from the thermosensitive liposomes. MRI relaxation parameters, $R_{1}$ and $R_{2}$, were measured at room temperature. The temporal variation in $R_{1}$ was used to obtain the temporal profile of cargo release. Due to their similar sizes, both the gadolinium and cargo (model cancer drug fluorescein) would come out of the liposomes together as a result of heating. The temporal variation in $R_{2}$ was used to monitor SPIO nanoparticles to enhance the tumor contrast. Monte Carlo spin dynamics simulations were performed by solving the Bloch equations and modeling SPIO nanoparticles as magnetized impenetrable spheres.

Results: TEM images and DLS measurements showed the diameter of the liposome nanoparticle $\sim 200 \mathrm{~nm}$. AMF heating showed effective release of the model drug. It was found that $R_{1}$ increased linearly by about $70 \%$ and then saturated as the cargo release process was completed, while $R_{2}$ remained approximately constant with an initial 7\%-drop and then recovered. The linear increase in $R_{1}$ is consistent with the expected linear cargo release with time upon AMF heating. Monte Carlo spin dynamics simulations suggest that the initial temporal fluctuation of $R_{2}$ is due to the plausible changes of SPIO aggregation and the slow non-recoverable degradation of liposomal membrane that increases water permeability with time by the heating process. The simulations show an order of magnitude increase in $\mathrm{R}_{2}$ at higher water permeability.

Conclusion: We have performed MR parameter study of the release of a cargo (model cancer drug, fluorescein) by magnetic heating from thermosensitive multifunctional liposomes loaded with dual contrast agents. The size of the liposome nanoparticles loaded with model cancer drug (fluorescein), gadolinium chelate, and SPIO nanoparticles was appropriate for a variety of cancer therapies. A careful and detailed analysis with theoretical explanation and simulation was carried out to investigate the correlation between MRI relaxation parameters, $R_{1}$ and $R_{2}$, and different cargo release fractions. We have quantified the cargo release using $R_{1}$, which shows a linear relation between each other. This result provides a strong basis for the dosage control of drug delivered. On the other hand, the fairly stable $R_{2}$ with almost constant value suggests that it could be used to monitor the position and condition of the liposomal site, as SPIO nanoparticles mostly remained in the aqueous core of the liposome. Because our synthesized SPIO-encapsulated liposomes could be targeted to tumor site passively by the EPR effect, or actively through magnetofection, this study provides a solid ground for developing $M R$ cancer theranostics in combination of this nanostructure and AMF heating strategy. Furthermore, our simulation results predict a sharp increase in $\mathrm{R}_{2}$ during the AMF heating, which opens up the exciting possibility of high-resolution, high-contrast real-time imaging of the liposomal site during the drug release process, provided AMF heating could be incorporated into an MRI setup. Our use of the clinically approved materials, along with confirmation by theoretical simulations, make this technique a promising candidate for translational MR cancer theranostics.
\end{abstract}

Key words: magnetic resonance theranostics, magnetic hyperthermia, alternating magnetic field (AMF), AMF-controlled drug release, thermosensitive multifunctional liposome 


\section{Introduction}

Cancer is the second highest leading cause of death worldwide, after cardiovascular diseases. Recently developed cancer theranostic approaches provide a promising direction to detect and treat cancer simultaneously [1-6]. This kind of molecular therapeutics, coupled with biomedical imaging, enhances the scope and efficacy of the treatment. For example, one of the major challenges to treat cancer by chemotherapy is to deliver the required high dose at tumor sites while minimizing the toxic effects on the benign tissues. Nanoparticle-mediated targeted and controlled drug delivery along with real-time imaging could achieve the desired result by reducing harmful effects on the benign tissues.

Nanovehicles of optimum size, functionality to target tumor cells, and capability to carry both the drug and contrast agent and deliver drugs at the tumor site under external signaling are required for such a theranostic procedure [7-9]. Various kinds of nanovehicles have been developed recently for the purpose of targeted delivery and controlled drug release [7-15]. Among them, promising candidates are liposomes [16-22] that have shown appealing features for drug delivery, biocompatibility, and clinical efficiency. Pharmacokinetic properties of the drug are significantly altered after encapsulation in the liposome and the toxicity of the drug is substantially reduced [23]. Furthermore, the drug is restricted from early activation during the circulation process. Different formulations of liposomes are now in clinical trial or already clinically approved [23-31]. They have been shown to be effective to decrease the off-target toxicity on other tissues. For example, liposomal anthracycline delivery reduces cardiotoxicity [23]. However, no significant increase in the therapeutic efficacy has so far been found in the clinical trials [23].

To alleviate this problem, liposome extravasation and bioavailability could be increased with the use of mild hyperthermia $\left(39-42^{\circ} \mathrm{C}\right)[23,32-35]$. Moreover, by increasing the concentration of drug at the tumor site through the leaky vascular permeability, chemotherapy at a slightly higher temperature has shown improved efficacy of the drugs. It has been found that a series of drugs show improved efficiency by heat activation. The synergistic effect of hyperthermia and chemotherapy are far more effective than the monotherapies [36-40]. The enhanced efficiency of the drugs has been attributed to the higher level of tumor perfusion, resulting in increased sensitivity to the tumor area [23]. Recent studies also showed that under mild hyperthermia, heat shock proteins released during cell necrosis acted as a trigger for antitumor immunity, thus regressing the tumor and reducing metastasis [41]. Hence, a combination of mild hyperthermia and significantly increased amount of drug delivery at the tumor site while reducing any interaction of the drugs with the healthy tissues along with simultaneous monitoring of the drug release and tumor site would be a vastly improved form of chemotherapy that could considerably improve the efficiency of the treatment.

Hyperthermia can be created by different methods such as a water bath [34], high intensity focused ultrasound (HIFU) [42-44], and alternating magnetic field (AMF) induced heat [45-48]. However, water baths could not provide spatially accurate treatment and HIFU is restricted in its ability for deep thermal therapy to a large area or penetrating bone and air. Here, the use of AMF to create mild hyperthermia has the advantages of achieving high accuracy in a specific area along with higher penetration capability, which is an alternative approach for disrupting the blood brain barrier [49]. Most importantly, as an ongoing project in our group, the AMF facility could be integrated into the existing MRI instrumentation and radio-frequency amplifiers for MR theranostics to simultaneously treat the cancer by using AMF and monitor the cancer treating process.

To complete such a drug delivery scheme, it is essential to have an efficient real-time monitoring system to observe and control the drug release at the target site [50-53]. MRI is a powerful noninvasive imaging modality in this regard, which has no harmful radiation and has general clinical acceptance. Previous studies have shown the single-modal MRI-guided drug delivery systems $[34,43,54,55]$. However, it is important to monitor simultaneously both the drug release and tumor site for an effective therapy. Dual-modal imaging is recommended over single-modal, as the latter could provide more handle to the clinicians to optimize the personalized treatment for better point-of-care. Combination of other imaging modalities might increase the scope of such treatments [56-58]. However, it is often restricted by high-energy ionizing radiation or limited penetration through the body. Hence, dual-modality within the scope of MRI could eliminate such restrictions for a successful treatment.

In clinical settings, clinicians mainly rely on either positive contrast $\mathrm{T}_{1}$-weighted imaging or negative contrast $T_{2}$-weighted imaging. However, a combination of both could provide insights on both the pathological phenomena and soft tissue anatomy to improve MR cancer imaging for stage detection, early diagnosis, and vascular imaging [59]. Therefore, many studies have turned to the design of encapsulating dual-modal MRI contrast agents in different formulations of liposomes and other nanocarriers [44, 60-68]. For example, Bos and coworkers [44] have shown the encapsulation of superparamagnetic iron oxide (SPIO) nanoparticles and Gd-chelate (ProHance) in liposomes for dual 
modal MRI. They have demonstrated in vivo HIFU treatment and discussed corresponding relaxation parameter changes. Other studies include different formulation of dual-modal MR contrast agents, such as synthesis of gadolinium and iron oxideconjugated nanoparticles [61], surface functionalization of SPIO-encapsulated liposome with gadolinium chelates [64, 67], size-controlled iron oxide nanoparticles [63] etc. Novel nanoparticle formulations with iron and manganese were developed for dual modal imaging and future theranostic purposes [68].

In this work, we have encapsulated SPIO nanoparticles, diethylenetriaminepentaacetic acid gadolinium(III) dihydrogen salt hydrate (Gd(III)DTPA, commercially known as Magnevist) molecules, and a commonly used small molecule model cancer drug (a fluorophore tracer, fluorescein $[48,69-71])$ into the thermosensitive liposome nanoparticles. We have demonstrated real-time AMF-controlled fluorescein release under mild hyperthemia and done a careful characterization of dual MRI parameters -- longitudinal relaxation rate $\left(R_{1}\right)$ and transverse relaxation rate $\left(R_{2}\right)$ with different amounts of cargo (fluorescein) release, as shown schematically in Figure 1. Gd(III)-DTPA molecules are released simultaneously with the cargo (fluorescein), changing $\mathrm{R}_{1}$ and making it sensitive to the percentage of cargo release. $R_{2}$ parameter monitors SPIO nanoparticles. We have done detailed analysis and explained the correlation between the relaxation parameters and cargo release with mathematical modelling and Monte Carlo spin dynamics simulations. Synthesized liposomes could be efficiently targeted to specific tumor sites through magnetic force $[72,73]$ or active targeting $[16,74]$. Hence, this work serves as a proof of concept for future MR theranostic approach, which could simultaneously fulfill all three important needs: 1) on demand cargo release using AMF-controlled mild hyperthermia; 2) monitoring

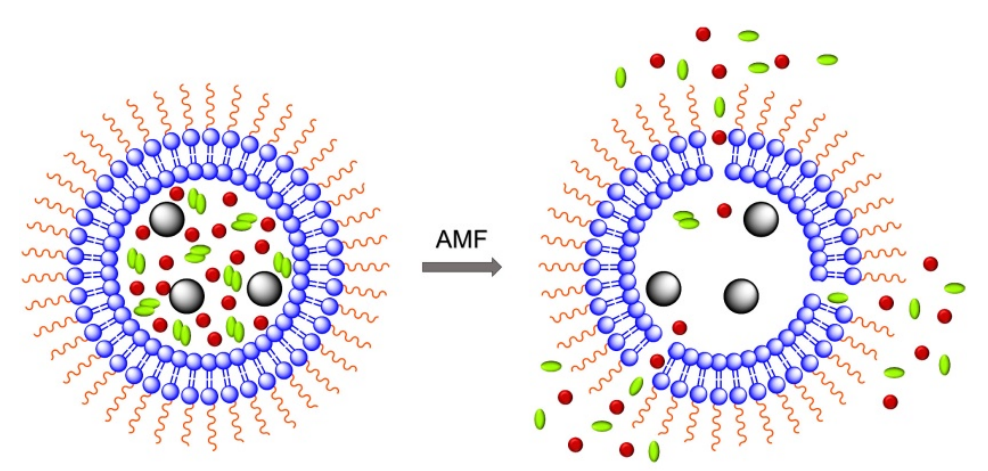

$\begin{array}{lll}\sim \sim & \text { Polyethylene glycol } & \text { Fluorescein (cargo/model drug) } \\ \text { Superparamagnetic iron oxide } & - & \text { Magnevist (Gd-DTPA) }\end{array}$

Figure 1. A schematic diagram of model drug/cargo (fluorescein) and contrast agents (magnetic nanoparticles and Gd(III)-DTPA) released from thermosensitive PEGylated liposomes under mild hyperthemia with alternating magnetic field (AMF). It illustrates the liposomes membrane permeability change and release of Gd(III)-DTPA and fluorescein upon AMF-induced heating. the position and condition of liposomal accumulation site (such as tumor) throughout the measurement by monitoring $\mathrm{R}_{2} ; 3$ ) dosage control of the cargo release process by monitoring $R_{1}$ parameter. Although we demonstrated our method using a model drug, existing in vivo and in vitro cancer drug release data and the associated diffusion models $[75,76]$ provide a strong basis for the validity of our results for real cancer drug release in animal body.

Here, we have used clinically approved formulations, so it has the potential to get readily adapted with magnetofection or any existing targeting approach. Theoretical simulations were carried out to understand the dynamical process during the AMF heating and a qualitative confirmation has been reached. The expected sharp rise in $\mathrm{R}_{2}$ with slightly increased water permeability of liposome, as seen from the simulations, indicates the possibility of high-resolution, high-contrast MR imaging of the liposomal site in in situ measurements.

\section{Materials and Methods}

\section{Preparation of hydrating solution}

Dextran-coated iron oxide nanoparticles of diameter $5-10 \mathrm{~nm}$ with $10 \mathrm{mg} / \mathrm{ml}$ concentration were bought from Ocean Nanotech, USA. $0.5 \mathrm{ml}$ of iron oxide solution was added to $1.5 \mathrm{ml}$ of phosphate buffer saline (PBS, $100 \mathrm{mM}$ ) (Sigma Aldrich) containing Gd (III)-DTPA (Sigma Aldrich) and fluorescein (Sigma Aldrich) to obtain a $2 \mathrm{ml}$ hydrating solution with a final concentration of 200 $\mathrm{mM} \mathrm{Gd}$ (III)-DTPA and $100 \mathrm{mM}$ fluorescein at $\mathrm{pH}$ 7.4 .

\section{Synthesis of liposome nanoparticles}

A total of 40 micromole of 1,2-dipalmitoyl-snglycero-3-phosphocholine (DPPC) (Avanti Polar Lipids, Inc.), 1,2-distearoyl-sn-glycero-3-phosphocholine (DSPC) (Corden Pharma), Cholesterol (Sigma Aldrich), and 1,2-distearoylsn-glycero-3-phosphoethanolamine-N-[ami no(polyethylene-glycol)-2000] (DSPE-PEG( 2000)) (Corden Pharma) in a molar ratio of 67:15:13:5 were dissolved in chloroform/ methanol (2:1) (Sigma Aldrich) mixture to yield an uniform solution of density between $10-20 \mathrm{mg} / \mathrm{ml}$. The homogeneous solution was evaporated under high vacuum at $40^{\circ} \mathrm{C}$ and kept for 5 hours in a rotatory evaporator for complete evaporation of the organic solvent. The produced thin film was hydrated with hydrating solution containing iron oxide nanoparticles, Gd(III)-DTPA, and fluorescein at pH 7.4 for 1 hour in a rotatory evaporator at a constant speed at $65^{\circ} \mathrm{C}$. The resulting solution was passed through $400 \mathrm{~nm}$ (31 
times) and $100 \mathrm{~nm}$ (51 times) filters, respectively, during extrusion using a mini extruder (Avanti Polar Lipid, Inc.). Non-entrapped iron oxide nanoparticles, Gd(III)-DTPA, and fluorescein were removed by repeated washing using Sephadex G-25M PD-10 (Sigma Aldrich). The liposome solution was further purified by repeated filtration through $0.1 \mu \mathrm{m}$ Amicon low-binding Durapore PVDF membrane (Millipore Corporation, Bedford, MA) at a centrifuge speed of $2000 \mathrm{rpm}$.

\section{Material characterization}

The size and zeta potential analysis were performed by dynamic light scattering (DLS) using a Zetasizer Nano (Malvern Instruments Ltd., Worcestershire, U.K.). The sizes of the liposome nanoparticles were measured before and after heating in $100 \mathrm{mM}$ PBS buffer. Zeta potential of the liposome solution was recorded in the deionized water. The morphology, distribution, and size of liposome nanoparticles were determined by transmission electron cryomicroscopy (CryoTEM). Grids were made by taking $2.5 \mu \mathrm{l}$ of the liposome sample on a glow- discharged Quantifoil holey-carbon grid (SPI Quantifoil R1.2/1.3) [77]. The grids were subsequently blotted dry and about 100 $\mathrm{nm}$ thick residual film of suspended solution across the holes in the grids was obtained by using a manual plunger. This solution was then rapidly plunged frozen around $-196^{\circ} \mathrm{C}$ into a 2:1 mixture of liquid propane: liquid ethane to produce a vitrified glassy solution within the holes. This quick freezing produced an amorphous ice-containing sample in the holy carbon film. The grid was then loaded with Gatan cryo specimen holder into FEI Titan Krios (Electron Imaging Center for NanoMachines, California NanoSystems Institute) microscope for imaging. The images were acquired by operating the machine at $200 \mathrm{kV}$ with a TIETZ F415MP 16 megapixel CCD camera.

The concentration of iron (Fe) or Gadolinium $(\mathrm{Gd})$ were quantitatively determined by inductively coupled plasma optical emission spectrometry (ICPOES) using a Shimadzu ICPE-9000 instrument. Different concentrations of liposome samples and supernatant solution were digested overnight with a $10 \mathrm{~mL}$ of aqua regia at $95^{\circ} \mathrm{C}$. Afterward, the solution was diluted with a $2 \%$ of $\mathrm{HNO}_{3}$ solution for quantitative measurement. The calibration curves for Fe or Gd were obtained from $0 \mathrm{ppm}$ to $10 \mathrm{ppm}$ and the results were fitted to obtain $\mathrm{Fe}$ and $\mathrm{Gd}$ amounts.

\section{AMF-controlled cargo (model drug fluorescein) release}

The set up was the same as described in the previous studies [47, 48]. Superparamagnetic heating was executed using a Magnetic Hyperthermia System manufactured by MSI Automation Inc. A five-turn copper coil (both the height and diameter $=50 \mathrm{~mm}$ ) was used for the experiment. The magnetic field oscillation frequency, amplitude, and induction power were $375 \mathrm{kHz}, 20 \mathrm{kA} / \mathrm{m}$, and $5 \mathrm{~kW}$, respectively. The fluorescence spectra were acquired using an Acton Spectra Pro 2300i CCD cooled below $-120^{\circ} \mathrm{C}$ with liquid nitrogen. CUBE 445-40C laser (Coherent Inc., Santa Clara, CA, USA) was used for excitation at a wavelength of $448 \mathrm{~nm}$ and a power of $4 \mathrm{~mW}$. Scattered and stray light beams were blocked with a long pass filter.

Synthesized liposome solution was diluted 10 times with $100 \mathrm{mM}$ PBS buffer. $1 \mathrm{ml}$ of the solution was exposed to AMF for 105 minutes. The fluorescence yield of fluorescein was measured after cooling the sample to room temperature at the end of each 15 minutes of AMF induced heating cycle. AMF heating would continuously increase the bulk temperature of the sample. The bulk temperature of the sample was measured using a thermometer at the end of each AMF induced 15-minute heating cycle. Thus, the measured temperature is the maximum temperature achieved at the end of the cycle. Each time, $50 \mu \mathrm{L}$ of the sample was added to $3.5 \mathrm{ml}$ of PBS buffer $(100 \mathrm{mM})$ in a standard $1 \mathrm{~cm}$ fluorescence cuvette. The intensity around the fluorescein emission maximum was integrated over the wavelength range from $510 \mathrm{~nm}-520 \mathrm{~nm}$ for three scans. The process was repeated three times and averaged over three such integrated emission spectra. The errors were computed from the standard deviation of three measurements. The fluorescence spectra of PBS were recorded in an identical way and used as the background for all the analyses.

In order to determine the percentage of cargo release as a result of AMF heating, the sample was completely lysed with a solution of $60 \mu \mathrm{L}$ of $10 \%$ triton X-100 and fluorescence spectra were acquired. The fluorescence spectra were acquired for $10 \%$ Triton added PBS buffer and a background spectrum for triton was obtained without adding the sample to the triton solution. The percentage of cargo release in each case was calculated according to the equation [45]:

$$
\text { Cargo release }(\%)=\left(\frac{\text { Flourescence of AMF treated sample }- \text { Fluorescence before AMF treatment }}{\text { Fluorescence after lysing with Triton }- \text { Fluorescence of Triton Background }}\right)
$$




\section{Tracking MRI parameters}

The samples with different cargo release were collected and the relaxation rates were measured using a $600 \mathrm{MHz}$ (AV600, Bruker) Nuclear Magnetic Resonance (NMR) spectrometer and micro-imaging setup. The relaxation rates of the sample after complete lysing by Triton X-100 were also measured. $\mathrm{R}_{1}$ was measured using the saturation recovery pulse sequence and $R_{2}$ was measured by the CPMG (Carr-Purcell-Meiboom-Gill) pulse sequence. The relaxation delay time was varied from 5-15 s, depending on the samples and the number of scans was $=1$. The saturation time was $300 \mathrm{~ms}$ and time of recovery was ranging from $5 \mathrm{~ms}$ to $10 \mathrm{~s}$ for saturation recovery pulse sequence. For CPMG pulse sequence, half of the interval between successive $180^{\circ}$ pulses $\left(\tau_{C P}\right)$ was $=500 \mu$ and time of echo (TE) was ranging from 2 to $100 \mathrm{~ms}$. Each measurement was repeated three times and the average value was taken. The errors were calculated from the standard deviation of the three measurements. All measurements were performed at room temperature.

The background relaxation rates were measured from the supernatant obtained after precipitating all the liposomes by centrifugation $(20,000 \mathrm{~g})$. The supernatant had been checked with TEM and DLS to ensure it was free from liposomes. The background correction was done on the relaxation rate and the corrected relaxation rates were determined for each sample. Percentage change in the relaxation rates were calculated as follows:

Change in Relaxation rate $(\%)=$

$\{($ Sample relaxation rate - Background relaxation rate $)-$ (Initial relaxation rate before heating - Background relaxation rate)\} Initial relaxation rate before heating - Background relaxation rate $\times 100 \%$

Longitudinal relaxivity $\left(\mathrm{r}_{1}\right)$ and transverse relaxivity $\left(\mathrm{r}_{2}\right)$ were deduced from the measurement of relaxation rates at different dilutions before and after completing magnetic heating and corrected by the background relaxation rate as follows:

\section{Corrected relaxation rate $=$ Sample relaxation rate - Background relaxation rate}

Corrected $\mathrm{Fe}$ and $\mathrm{Gd}$ concentrations were obtained from ICP-OES measurements according to the equation:

\section{Corrected concentration $=$ Sample concentration - Background concentration}

The corrected relaxation rates were plotted with the corrected concentration and a linear fit was obtained. The relaxivity parameter was obtained from the slope of the fitted straight line.

\section{Monte Carlo spin dynamics simulations}

Monte Carlo spin dynamics simulations using a diffusion model were performed to calculate $R_{2}$ parameter for different inter-nanoparticle separation of SPIO aggregations and water permeability of liposome membrane. SPIO nanoparticles were modeled as magnetized impenetrable spheres of radius $=5 \mathrm{~nm}$. Based on cryoTEM images, we estimated that there were 7 SPIO nanoparticles inside each $100 \mathrm{~nm}$ radius liposome. Inter-nanoparticle distances were taken as 2, 6, and 10 times of SPIO nanoparticle radius to simulate different degree of aggregations. The root mean square (rms) angular frequency shift at the nanoparticle surface (compared to a point infinitely far away) was taken as $\Delta \omega_{\mathrm{r}}=1.7 \times 10^{7} \mathrm{rad} / \mathrm{s}$. The diffusing water magnetizations were first positioned stochastically and the diffusion was modeled by random walks with periodic boundary conditions. The diffusion coefficient was taken as $2.3 \times 10^{-9} \mathrm{~m}^{2} / \mathrm{s}$ for water at $25^{\circ} \mathrm{C}$. In the simulation process, the effect of liposome membrane permeability was included as the probability of a diffusing water magnetization striking the liposome membrane to cross the barrier and enter inside the liposome. After each random-walk step, each water magnetization experienced a new, combined, net magnetic field, which was calculated by adding the dipolar fields from all the magnetic nanoparticles present. The Z-component of the induced dipolar field, $B_{d i p, Z}$, at position $(d, \theta)$ form the magnetic nanoparticle could be approximated by:

$$
B_{d i p, z}(d, \theta)=\sqrt{5 / 4} \frac{r^{3} \Delta \omega_{r}}{\gamma d^{3}}\left(3 \cos ^{2} \theta-1\right)
$$

where $\gamma$ is the gyromagnetic ratio of ${ }^{1} \mathrm{H}, r$ is the radius of the magnetic nanoparticle, $\Delta \omega_{\mathrm{r}}$ is the root mean square (rms) angular frequency shift at the magnetic nanoparticle surface, $d$ is the distance from the point to the center of the magnetic nanoparticle, and $\theta$ is the angle between the $Z$-axis and the position vector of the point.

Following an initial $90^{\circ}+y$ excitation pulse that flips all the equilibrium water magnetizations from $+\mathrm{z}$ to the $+\mathrm{x}$ direction, the time evolutions of 1000 diffusing water magnetizations were calculated by numerical integration of the Bloch equations [78] by Matlab (The MathWorks, Inc., Natick, MA, USA) using ordinary differential equation (ODE) solvers. As in the actual experiments, $\tau_{C P}$ in the CPMG pulse sequence was taken as $500 \mu \mathrm{s}$. The average net water magnetization was calculated by averaging all the individual water magnetizations. The CPMG $\mathrm{T}_{2}$ relaxation rate $\left(R_{2}\right)$ was obtained by a linear fitting of the negative natural logarithm of the normalized transverse average water magnetization as a function of time. 


\section{Results and Discussions}

\section{Material characterization}

The DLS results in PBS buffer provide an effective diameter of $231 \mathrm{~nm}$ for liposome nanoparticles with polydispersity index $=0.134$. Intensity profile for size distribution has been shown in Figure 2A. It suggests that the nanoparticles are stable and well dispersed under biological condition at $\mathrm{pH}$ 7.4. DLS measurements were repeated after AMF heating and similar sizes (diameter $=223 \mathrm{~nm}+/-1.6 \mathrm{~nm}$ ) of liposome nanoparticles with polydispersity $=0.178$ were obtained. Zeta potential of the liposome nanoparticles measured in water was found to be $-12.63 \mathrm{mV}$. CryoTEM results are shown in the left and right panels of Figure 2B in different magnifications. They show homogeneous distribution and morphology of liposomes with embedded SPIO nanoparticles. Electron-dense objects, such as SPIO, appear as darker regions in the image. Larger aggregates of SPIO were visible as dark spots at this resolution. The magnified one in inset of the right panel demonstrates the encapsulation of SPIO nanoparticles inside the inner aqueous core of the liposomes. The $\mathrm{Gd}$ and $\mathrm{Fe}$ concentrations in the liposome solution were determined by ICP-OES to be $1.37 \mathrm{mM}$ and $22.2 \mathrm{mM}$ with $\sim 5 \%$ uncertainty, respectively. Considering the unencapsulated $0.00382 \mathrm{mM} \mathrm{Gd}$ and $6.50 \mathrm{mM}$ SPIO in the supernatant, the effective $\mathrm{Fe}$ and $\mathrm{Gd}$ concentrations in the liposome solution are $15.7 \mathrm{mM}$ and $1.37 \mathrm{mM}$, respectively.

The synthesized liposomes with uniform size distribution (diameter $\approx 200 \mathrm{~nm}$ ) and low polydispersity $(<0.2)$ indicate a homogeneous distribution in the solution. CryoTEM images demonstrate the successful encapsulation of SPIO nanoparticles inside the liposome nanoparticles.
The optimal size of the encapsulating liposome nanoparticle plays a key role in this kind of theranostic approach. Smaller size like less than 5 $\mathrm{nm}$ accelerates fast excretion through kidney filtration, while larger-size nanoparticles are easily recognized for uptake by reticuloendothelial system (RES). A size of around the inter-endothelial cell gap of a few hundred nanometers is considered as a key factor for the determination of optimal size, and previous studies have shown preferred size range is about $50-200 \mathrm{~nm}[79,80]$ to reduce fast clearance and enhance passive tumor targeting by enhanced permeability and retention (EPR) effect [81].

SPIO-encapsulated liposomes are ideal candidates for magnetic targeting to the tumor and recent in vivo studies have shown the nanocarriers in the range of $200 \mathrm{~nm}$ could be efficiently targeted to the tumor by magnetofection $[17,72,73]$. In order to maximize the hyperthermia effect and magnetic targeting, a size of about $200 \mathrm{~nm}$ is generally preferred, as the magnetic force on the liposome would get enhanced in proportion to its size [17]. The association of polyethylene glycol (PEG) with the liposome nanoparticles is to increase the circulation time in blood for passive targeting by EPR. The unaltered size and polydispersity index before and after the AMF heating suggest that the liposome structure remained intact and the SPIO nanoparticles remained inside the liposome throughout the procedure. Additionally, the presence of SPIO nanoparticles inside the liposomes offers the nanovehicles active tumor targeting capability for superficial tumors by external magnetic force [72, 73]. Consequently, SPIO-encapsulated liposomes are structurally suitable to make nanoparticles biocompatible and offer a clinically proven, versatile platform for the further enhancement of pharmacological efficacy and targeting efficiency.
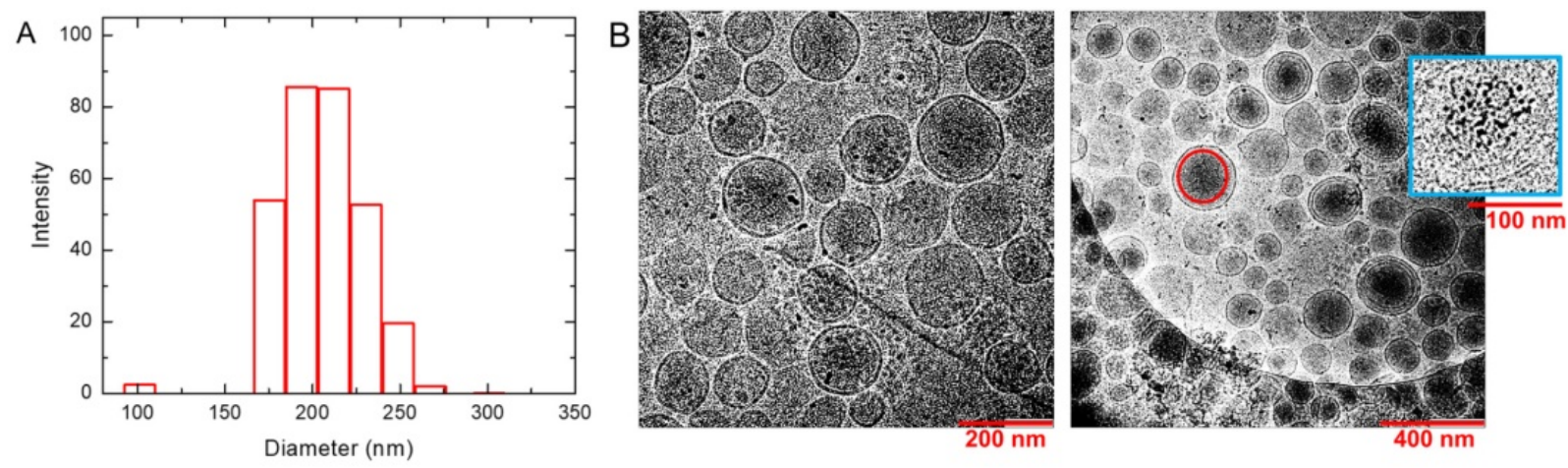

Figure 2. Characterization of liposomes. (A) Dynamic light scattering (DLS) of synthesized liposomes. It demonstrates the distribution of nanoparticles with an average diameter $231 \mathrm{~nm}$ and polydispersity 0.134. (B) CryoTEM images show the homogeneous distribution and morphology of liposomes in different magnifications. They show an average diameter around $200 \mathrm{~nm}$ and successful formation of bilayered spherical liposomes. The darker regions mark the presence of SPIO inside the liposome. A representative red circle is drawn to show such an SPIO concentrated region. The magnified liposome in blue box of the right panel shows the SPIO encapsulation inside the aqueous core of liposomes. Larger aggregates of SPIO appeared as darker spots. 


\section{AMF-controlled cargo (model drug fluorescein) release}

The cargo release profile following AMF heating was monitored by using the self-quenching property of fluorescein [69, 71] that serves as a model for cancer drug, as it has the similar molecular size as the real cancer drug. The fluorescence yield is quenched depending on whether the fluorescein is inside the liposome with a high concentration or outside the liposome with a lower concentration. This property was used to quantify the percentage of fluorescein release from the liposome under AMF heating. All fluorescence measurements were performed after cooling the sample to room temperature following AMF heating. Although the measurements were performed up to a maximum bulk temperature of $39^{\circ} \mathrm{C}$, the local temperatures inside liposomes could be higher than the bulk temperature by $15^{\circ} \mathrm{C}-20^{\circ} \mathrm{C}$ [82]. We have checked that the fluorescence properties remained unchanged up to $80^{\circ} \mathrm{C}$ by heating fluorescein up to $80^{\circ} \mathrm{C}$ in a water bath and then performing fluorescence experiments after cooling it to room temperature. No change in the spectral characteristics of fluorescein was seen in these experiments.

The characteristic cargo (fluorescein) release pattern as measured by fluorescence yield is shown by the dotted red curve in Figure 3A. We find from the dotted red curve in Figure $\mathbf{3 A}$ that the percentage of cargo release was initially low, and after a threshold, it increased at a faster rate and finally reached the maximum, indicating complete cargo release. The CryoTEM images in Figure 2B show that the number of SPIO nanoparticles in each liposomes varies, leading to the conjecture that the higher loaded liposomes released cargo at the beginning, and later on, other liposomes with average loading started to release cargo. Figure 3A and Figure $3 \mathrm{~B}$ show experimental points for cumulative AMF heating time and corresponding maximum temperature of the bulk sample (before cooling). Figure 3A (dotted red curve) shows that the cargo release rate is fastest in the $(30-45)$ minute time interval and levels off after an hour, indicating complete cargo release. However, the exact timing depends on the circumstantial set up and needs to be optimized, based on the clinical settings. A control liposome sample was measured before and after the treatment procedure to ensure that there was no leakage otherwise.

The dotted black line in Figure 3A represents the spontaneous cargo release profile of liposomes, which clearly shows cargo was not leaked without AMF heating. Figure 3B shows profile of the corresponding bulk temperature change with cargo release. The saturation temperature for the drug release is $37^{\circ} \mathrm{C}$ and the dramatic increase in the rate of drug release occurred between $34^{\circ} \mathrm{C}-37^{\circ} \mathrm{C}$ resulting in almost $100 \%$ drug release. AMF heating experiments show that significant percentage of drug release starts above $35^{\circ} \mathrm{C}$ and saturates at $37^{\circ} \mathrm{C}$ for this liposome. Figure $\mathrm{S} 1$ in supplementary material shows a comparison of cargo release at different temperatures under AMF heating (red bar) and bulk heating in a bath (black bar). We find a significantly higher percentage of drug release at around $36^{\circ} \mathrm{C}$ under AMF heating, compared to that under the bulk bath heating. The choice of liposome provides the flexibility to easily manipulate the transition temperature by changing the lipid composition according to the clinical requirement [83].

Measuring local temperature inside the nanovehicle is challenging. However, there have been several methods to measure temperature inside the core of liposome by optical spectroscopy or using polymers [82, 84-87]. Ongoing effort in our labs is to develop theoretical methods to account for the aggregate formation and the size distribution of the magnetic nanoparticles inside the core of liposome to accurately estimate the specific loss power and heating efficiency for MR nanotheranostic hyperthermia in cancer therapy [35]. Based on those studies, generally speaking, higher the number of SPIO nanoparticles in the core of the liposome, more heat can be induced by the application of AMF.
Figure 3. Cumulative cargo (fluorescein) release profile. (A) Cumulative cargo (fluorescein) release profile is shown as a function of the cumulative AMF heating time. Dotted red curve demonstrates the percentage of cargo release at different time. The characteristic pattern indicates the initial slow rate of release, then an increased rate of release between 30 to 45 minutes, and finally leveling off, indicating the complete release. Dotted black curve shows the spontaneous release of cargo from liposomes without AMF treatment and flat line depicts no leakage is observed otherwise. (B) Cumulative cargo (fluorescein) release profile is shown as a function of the maximum bulk temperature (before cooling to room temperature). Fluorescence measurements were performed at room temperature. It demonstrates that the maximum change in the bulk temperature during the process from no cargo release to complete release is about $3^{\circ} \mathrm{C}$. The dotted lines through the data points are included in each case as a guide to the eye (see text for details). 


\section{Tracking MRI parameters}

Figure $4 \mathrm{~A}$ shows the percentage change of MRI parameter $R_{1}$ with the percentage of cargo release (dotted red line). We find a linear increase in the percentage of $R_{1}$ with the percentage of cargo release, and a maximum increase of $R_{1}$ is $69.8 \%$, indicates $100 \%$ cargo release. The results have been given in a tabular form in Table 1. This change in the $R_{1}$ value is attributed to the simultaneous release of Gd(III)-DTPA along with the model drug molecule fluorescein (cargo) from the liposome nanoparticles, since the presence of Gd(III)-DTPA outside the liposome would significantly increase the $R_{1}$ value of the water protons due to the free interaction of $\mathrm{Gd}(\mathrm{III})$ with the water protons, compared to those in the encapsulated state [34]. This hypothesis has been further confirmed by measuring the $\mathrm{R}_{1}$ value of the liposome solution after completely lysing with Triton X-100, which provides the exact same value, as found from the maximum $R_{1}$ value after complete cargo release. It implies that the local heating increases the permeability of liposome membrane [88, 89], allowing leakage of both Gd(III)-DTPA and cargo, and $R_{1}$ increases linearly with the cargo release. In order to further investigate the linear nature of the plot, we have fitted percentage changes in $R_{1}$ with percentage cargo (fluorescein) release in Figure 4B and obtained a linear equation (shown below) with squared correlation coefficient $\mathrm{R}^{2}=0.997$.

$$
\begin{gathered}
\% \text { Change in } \mathrm{R}_{1}=0.686 \times(\% \text { Change in cargo } \\
\text { release })
\end{gathered}
$$

Table 1. Data for percentage changes in MR relaxation rates with different percentage of cargo (fluorescein) release

\begin{tabular}{lll}
\hline \% Cargo (fluorescein) release & $\mathbf{~} \mathbf{\%}_{\mathbf{1}} \mathbf{R}_{\mathbf{1}}$ change $^{\mathbf{b}}$ & $\mathbf{\%} \mathbf{R}_{\mathbf{2}}$ change $^{\mathbf{b}}$ \\
\hline 0 & 0 & 0 \\
1.194 & -0.482 & -1.746 \\
6.202 & 4.373 & -7.548 \\
69.449 & 46.935 & -1.766 \\
85.559 & 58.833 & -2.195 \\
95.228 & 69.904 & 0.272 \\
101.739 & 65.780 & -1.878 \\
\hline
\end{tabular}

aStandard deviation is $3 \%$. bStandard deviation is $0.3 \%$. $\mathrm{R}_{1}$ : Longitudinal relxation rate, $\mathrm{R}_{2}$ : Transverse relaxation rate, MR: Magnetic resonance

The black curve in Figure $\mathbf{4 A}$ shows the percentage change of $R_{2}$, which remains essentially constant, independent of cargo release (data are
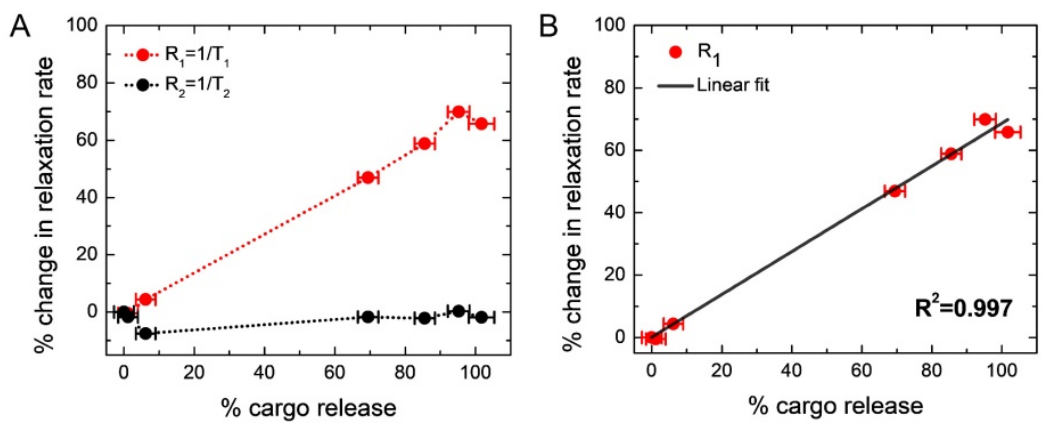

Figure 4. Correlation between changes in $M R$ relaxation rates and cargo (fluorescein) release. (A) Red curve indicates the percentage change in $R_{1}$ and black curve indicates the percentage change in $R_{2}$. It demonstrates $R_{1}$ linearly increases with cargo release, and shows maxima after $69.8 \%$ increase, when cargo release was complete. $R_{2}$ is held nearly constant; however, it shows an initial 7\%-drop (from the second to the third point, corresponding to the path of no cargo release to about $6 \%$ cargo release) and then a gradual increase to the initial value. Please see the text for the plausible explanation. The dotted lines through the data points are included in each case as a guide to the eye. (B) Fitted linear plot for percentage change in longitudinal relaxation rate $\left(R_{I}\right)$ with percentage cargo (fluorescein) release. The fitted linear equation: \% Change in $R_{I}=0.686 \times(\%$ Change in cargo release) with squared correlation coefficient $\mathrm{R}^{2}=0.997$.
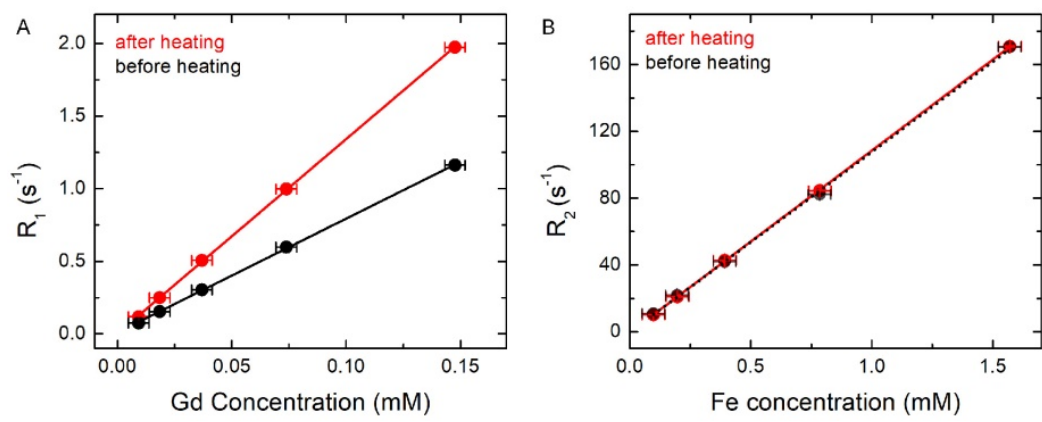

Figure 5. MR relaxation rates were measured at different dilutions before and after complete AMF heating. (A) $R_{1}$ versus $G d$ concentration before and after complete AMF heating. It shows the slope of the fitted linear plot before (black) $r_{l}=7.84 \mathrm{~s}^{-1} \mathrm{mM}^{-1}(\mathrm{Gd})$ and after complete AMF heating (red) $r_{1}=13.38 \mathrm{~s}^{-1} \mathrm{mM}^{-1}$ (Gd). (B) $R_{2}$ versus Fe concentration before and after complete AMF heating. It shows the slope of the fitted linear plot before (black) $r_{2}=108.01 \mathrm{~s}^{-1} \mathrm{mM}^{-1}$ $(\mathrm{Fe})$ and after complete AMF heating (red) $r_{2}=108.93 \mathrm{~s}^{-1} \mathrm{mM}^{-1}(\mathrm{Fe})$. The uncertainties on $\mathrm{R}_{1}$ and $\mathrm{R}_{2}$ are $<1 \%$, whereas the uncertainties on the concentration are $\sim 5 \%$. shown in Table 1). However, a closer look reveals that initially (along second to third point in the black curve in Figure 4A, corresponding to the path of no cargo release to about $6 \%$ cargo release or the $3^{\text {rd }}$ point in Table 1) it dropped by about $7 \%$ and later it increased back to the initial value. DLS measurements did not show any significant change of the size of liposomes as a result of mild AMF heating. On the other hand, $R_{2}$ parameter of the completely lysed sample (where all the SPIO nanoparticles should be outside the liposome encapsulation) was found to be reduced by $\approx 20 \%$, compared to that in the pretreated sample (data not shown). Hence, mild AMF heating is not causing any significant leakage of SPIO nanoparticles from the liposome encapsulation, as also concluded in earlier work [44]. Since SPIO particles remained inside liposome during AMF heating and the liposome could be attached to the tumor site, the encapsulated SPIO particles could be used for online monitoring of the tumor site. Our theoretical simulations provide a qualitative explanation of the initial small drop and later slight increase of $\mathrm{R}_{2}$ under mild AMF heating (Figure 4A), as discussed in the next section. 
In order to further understand and quantify the changes of the relaxation rates, $R_{1}$ and $R_{2}$, relaxivities $\left(r_{1}\right.$ and $\left.r_{2}\right)$ were determined by measuring the relaxation rates at different dilutions both before and after complete AMF heating. In Figure $5 \mathrm{~A}$, black and red lines show $\mathrm{R}_{1}$ versus $\mathrm{Gd}$ concentration plots before and after complete AMF heating, respectively. The slope of the black line (before AMF heating) is $r_{1}=7.84 \mathrm{~s}^{-1} \mathrm{mM}^{-1}$, while the slope of the red line (after AMF heating) is $r_{1}=13.38$ $\mathrm{s}^{-1} \mathrm{mM}^{-1}$. In Figure 5B, we show the plots of $R_{2}$ versus $\mathrm{Fe}$ concentration both before (in black) and after (in red) complete AMF heating. It shows that the slopes of the red and black lines are $r_{2}=108.93$ $\mathrm{s}^{-1} \mathrm{mM}^{-1}$ and $108.01 \mathrm{~s}^{-1} \mathrm{mM}^{-1}$, respectively, i.e. they are about equal. It is clear from the plots that AMF heating significantly affects the $R_{1}$ versus $G d$ concentration curve and increases $r_{1}$ of the AMF-treated sample by $\approx 70 \%$, whereas the heating has almost no effect on the $R_{2}$ versus $\mathrm{Fe}$ concentration curve. Furthermore, the complete linear nature of the plots in Figure 5 confirms that the changes in longitudinal relaxation rates, $R_{1}$, are mainly affected by $\mathrm{Gd}$ or Gd(III)-DTPA concentration, and changes in transverse relaxation rates, $R_{2}$, are associated with $\mathrm{Fe}$ or SPIO concentration.

\section{Monte Carlo spin dynamics simulations}

Figure 6A shows the effect of liposome membrane permeability on $R_{2}$ for different inter-nanoparticle distances $\left(\mathrm{D}_{\mathrm{pp}}\right)$ of the SPIO aggregates. Here, we define water permeability as the probability of a diffusing water magnetization striking the liposome membrane to cross the barrier and enter inside the liposome. We have plotted membrane permeability versus $R_{2}$ for different values of $D_{p p} / r_{p}$ (where $r_{p}$ is the radius of a SPIO nanoparticle). Higher $D_{p p}$ indicates weaker aggregation state of SPIO and smaller $\mathrm{D}_{\mathrm{pp}}$ implies stronger aggregation state. We find from Figure 6A that the nature of variation of $R_{2}$ with permeability
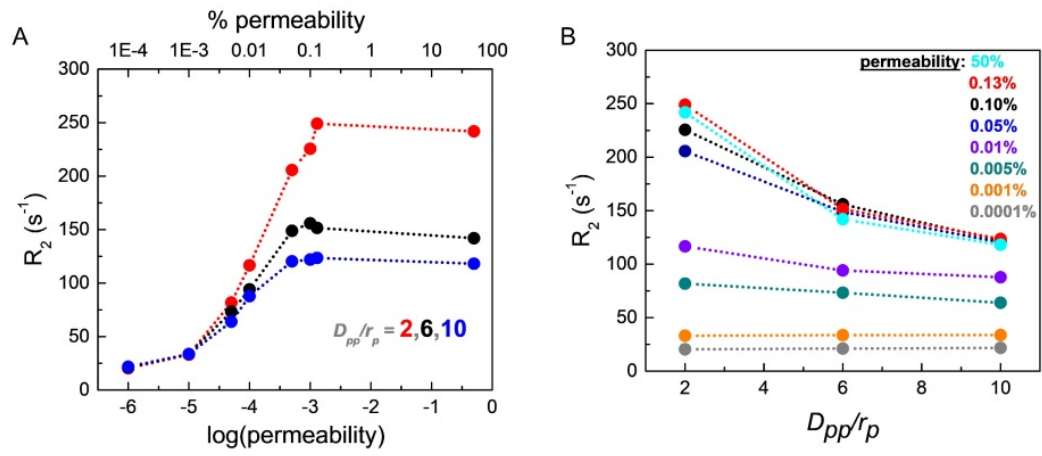

Figure 6. The effects of liposome membrane permeability and SPIO aggregation inside liposome on CPMG $\mathbf{R}_{2}$ relaxation rates. (A) CPMG relaxation rates $R_{2}$ are plotted as a function of the liposome membrane permeability at three different aggregation states with $D_{p p}$ (inter-SPIO nanoparticle distance) $/ r_{P}$ (single $S P I O$ radius) $=2,6$, and 10 , respectively. The radius of a single SPIO, $r_{p}=5 \mathrm{~nm}$. (B) CPMG relaxation rates $R_{2}$ are plotted as a function of the degree of aggregation, $\mathrm{Dpp} / \mathrm{r}_{\mathrm{p}}$, at different liposome membrane permeability conditions. The dotted lines through the points are included in each case as a guide to the eye. is qualitatively independent of $D_{p p} / r_{p}$. R $R_{2}$ increases sharply with permeability from $1 \times 10^{-3} \%$ to $0.1 \%$ and saturates for permeability $>0.1 \%$. Thus, even a small change in liposome membrane permeability in the range $\left(1 \times 10^{-3} \%\right.$ to $\left.0.1 \%\right)$ would result in a significant increase of $R_{2}$. As permeability increases, the water molecules are more likely to diffuse closer to the SPIO nanoparticles encapsulated inside the liposome and exchange faster with the "fresh" water magnetization outside the liposome, thus experiencing stronger fluctuating magnetic fields and resulting in a higher $R_{2}$ value, as seen in Figure 6A. Figure 6B shows the correlation between $R_{2}$ and $\mathrm{D}_{\mathrm{pp}} / \mathrm{r}_{\mathrm{p}}$ for different liposome membrane permeabilities. We find that the liposome membrane with permeability higher than $1 \times 10^{-3} \%$ exhibits a significant drop in $R_{2}$ value with higher $\mathrm{D}_{\mathrm{pp}}$. However, the liposomes with membrane permeability less than $1 \times 10^{-3} \%$ do not show noticeable change in $R_{2}$ for different aggregation status. The diffusing water magnetizations experience stronger dipolar magnetic fields when SPIO is in stronger aggregation state, compared to those in the weaker aggregation state where the resulting net SPIO dipolar field experienced by diffusing water magnetizations is partially cancelled out by individual fields [44, 54].

We have estimated the chance of a diffusing water magnetization passing through the intact liposome membrane per collision would be between $1 \times 10^{-5}$ and $1 \times 10^{-4}$ (or $0.001 \%-0.01 \%$ ) in normal condition $[90,91]$, taking the thickness of liposome membrane $\approx 3 \mathrm{~nm}$ and the water permeability $10^{-1.95}$ $\mathrm{cm} / \mathrm{s}$. Liposome membrane undergoes reversible permeability change on heating [88, 89], and this change is significantly higher near the phospholipid phase transition temperature of $\approx 41^{\circ} \mathrm{C}$ [44]. In this experiment, we did the heating close to its transition temperature, leading to the possibility of significant permeability change of the synthesized thermosensitive liposome membrane. However, the $\mathrm{R}_{2}$ measurements were done after cooling the sample to room temperature. Since the permeability change should be mostly reversible initially under mild heating, we do not expect any significant increase of the measured $R_{2}$ due to the change of membrane permeability at the initial stage. On the other hand, during the process of AMF heating, as the membrane permeability would increase, there would be higher access of water molecules inside the liposome, thus increasing $\mathrm{D}_{\mathrm{pp}}$ and causing disaggregate of SPIO clusters. This change of the SPIO aggregation state inside liposome should be retained when the solution 
was cooled back to room temperature and cause a slight decrease of $R_{2}$ value initially. As the heating process would continue repeatedly, the change in the liposome membrane permeability might not be completely reversible when cooled back to room temperature, causing $R_{2}$ value to rise. The interplay of these two opposing effects explains the observed initial small drop in $R_{2}$ and a subsequent slight increase, as seen in Figure 4A (black curve).

On the other hand, unencapsulated SPIO nanoparticles would be dispersed uniformly in the solution. Only a very small fraction can form aggregates under thermal equilibrium and the average value of $D_{p p} / r_{p}$ is expected to be higher for such aggregates in the absence of any physical constraints like liposome membrane to ensure that they remain close to one another. Hence, $R_{2}$ value should decrease if all the SPIO nanoparticles would come out from the liposome enclosure as a result of lysing with Triton X-100. Experimentally, we have observed $\approx 20 \%$ decrease in the $R_{2}$ value after lysing with Triton X-100 (data not shown) and this decrease is much higher than the initial 7\%-drop in $\mathrm{R}_{2}$ due to mild AMF heating. Since Figure $4 \mathrm{~A}$ does not show any large drop $(\approx 20 \%)$ in the $R_{2}$ value, we conclude that SPIO nanoparticles remain within the liposome enclosure during the mild AMF heating process.

We performed $R_{2}$ measurements at room temperature after cooling the sample, when the liposome membrane property should be largely restored. However, our simulation results (Figure 6A) predict a sharp increase in $R_{2}$ with small increase of the liposome membrane permeability. Therefore, we expect to observe significant increase in $\mathrm{R}_{2}$ for in situ MRI measurements with AMF heating. Since liposome could be efficiently functionalized to target the tumor site $[16,74]$, such in situ real-time MRI measurements with AMF heating opens up the possibility of high-resolution, high-contrast imaging of the tumor site during the MR theranostic process because of the large $R_{2}$ values.

In principle, the $r_{1}$ and $r_{2}$ relaxivities in spin systems with magnetic nanoparticles increase with temperature [92], as both diffusion of the water ${ }^{1} \mathrm{H}$ spins and nanoparticle cluster size increase with temperature [35]. In our current study, however, such temperature-dependent effect may be ignored compared with the dominating permeabilitydependent effect, due to the small temperature range used under mild hyperthermia condition and the fact that the magnetic nanoparticles are encapsulated inside the core of the liposome. On the other hand, the liposome membrane permeability changes significantly over the mild-hyperthermia temperature range, as the temperature above which the fluorescein drug release rate from this liposome increases rapidly is about $35^{\circ} \mathrm{C}$ (Figure 3B), although the phospholipid phase transition temperature for this liposome is $41^{\circ} \mathrm{C}$ [44].

\section{Future scope and application}

Compared to the previous studies done by Bos and coworkers [44], we have used liposomes with similar compositions; moreover, we have incorporated a model cancer drug fluorescein to study controlled dose release at target site. Furthermore, we have used AMF controlled delivery, which safely allows deeper penetration inside human body compared to HIFU and increases the scope of treatment. Earlier studies [37, 55 ] have shown that encapsulation of cancer drug inside temperature sensitive liposome increases tumor drug concentrations and improve antitumor efficacy of the drugs. The studies have shown 2-4 fold [40] and 2-16 fold [93] increase compared to non-heated condition, respectively. We have used a similar PEGylated temperature sensitive liposome and shown AMF controlled heating mechanism in relation to dosage delivery and carefully characterized MR relaxation parameters by encapsulating contrast agents at the same time. Hence, our study successfully extends previous studies to MR cancer theranostics. Moreover, at higher temperature, the permeability of liposome membrane increases. The simulations show that $R_{2}$ parameter increases rapidly with the permeability of the liposome membrane, raising the possibility of getting high-quality MRI images, if MR measurements could be done during AMF heating at the elevated temperature.

In this study, we have used cargo fluorescein as a model cancer drug and established a detailed correlation between the magnetic heating stimulated cargo releases with MR relaxation parameters through experimental study and theoretical understanding. Previous extensive studies were done $[75,76,94]$ to understand the rate of release of real cancer drugs at in vitro and in vivo conditions and the results have been well understood by different diffusion- and dissolutionbased mathematical models (squared correlation coefficient near 1), such as the Reciprocal Powered Time (RPT) or Weibull (W) model [75, 76]. Authors [76] found that in vitro and in vivo data could be best fitted in the RPT model, which is based on the general equation of dissolution and diffusion rate limited process $\mathrm{dw} / \mathrm{dt}=(\mathrm{D} / \mathrm{h}) \times \mathrm{S} \times \mathrm{C}_{\mathrm{s}}$ under sink condition, where $\mathrm{dw} / \mathrm{dt}$ is the rate of drug release, $\mathrm{D}$ is the drug molecule diffusion coefficient, $\mathrm{S}$ is the effective surface area of drug with release medium, $\mathrm{C}_{\mathrm{s}}$ is solubility of the drug, and $\mathrm{h}$ is the length of diffusion path. Other studies found a good linear correlation between drug release in in vitro experiments with the drug absorption in in vivo experiments [75]. Hence, it is clear that the rate of real cancer drug release primarily depends on 
diffusion and solubility. The diffusion coefficient depends on the hydrodynamic size of the drug molecule. It is well known that the hydrodynamic size of fluorescein is similar to many cancer drug molecules and therefore fluorescein was used as a model cancer drug in a large number of previous studies [48, 69-71].

Another important factor comes from the solubility of the drug, which depends on its particular nature, bonding, and hydrophobicity. For the poorly soluble cancer drugs, rate of release would be slower and, accordingly, AMF time could be adjusted depending on the exact clinical situation. However, the probability of encapsulation of a molecule in liposome is also proportional to the solubility of the molecule in the medium, and hence correspondingly fewer cancer drug molecules would be encapsulated. As a result, the AMF driven percentage release of drug, i.e., $[(\mathrm{dw} / \mathrm{dt}) / \mathrm{w} * 100]$, through diffusion of encapsulated molecule with different solubility would be similar. Hence, we expect qualitatively similar results for both model drug fluorescein and real cancer drug in in vitro or in vivo conditions, when the results are plotted as the percentage of drug release. Simultaneous release of Gd(III)-DTPA and fluorescein or the changes in aggregation status of SPIO are expected to remain unaltered for in vivo situation. Thus, the observed linear correlation between percentage drug release versus the percentage increase in $R_{1}$ can also be expected for real cancer drugs in clinical conditions, because the drug release process is essentially a diffusion process through a membrane. Similarly, the characteristic pattern of percentage changes in $R_{2}$ as a function of the percentage release of encapsulated drug would remain qualitatively the same for real cancer drugs in clinical condition. However, if the real drug would interact with the membrane of liposome or the process would not be diffusion, the results could be different. Nevertheless, for a large class of commonly used cancer drugs, the diffusion model holds and our results should remain valid for real cancer drugs in clinical conditions.

In this proof-of-concept study, we have focused on temperature effect on drug release. In a mild hyperthermia, blood perfusion and tumor oxygenation pattern change inhomogeneously and this change in local environment is very crucial for planning an effective treatment protocol. Various bioheat equations, tissue parameters, and temperature details should be taken into account to estimate tumor perfusion and changes in tumor local environment after employing mild heating. In this regard, many theoretical modeling and experiments were done [95-97] with real tumor. Future in vivo and clinical studies are required in this regard to implement the technique for clinical application. Systematic measurements of the drug release after heating with distinct temperature and time using a water bath and using AMF, respectively, would provide insights to the heating mechanism and the interplay between the local temperature and the bulk temperature.

\section{Conclusions}

Real-time visualization of nano-drug carrier biodistributions, drug release processes, and therapeutic responses could provide critical information to dynamically optimize treatment operations in precision medicine in real time. In this work, we have demonstrated a novel MR theranostic approach that could be combined with the established targeting capacity of liposomes to deliver and release cargo with spatial control by AMF heating and simultaneously monitor both the cargo release and condition of the liposomal sites. We have used appropriate size of liposome nanoparticles and shown that the cargo release from the liposomes by AMF controlled mild hyperthermia could be quantified by measuring the longitudinal relaxation rate, $R_{1}$. We have confirmed that SPIO remained inside the liposome enclosure during AMF heating by observing that $R_{2}$ remained approximately constant during the heating process. Monte Carlo spin dynamics simulations provided qualitative explanations to the observed variations in $R_{2}$ during the $\mathrm{AMF}$ heating process and predicted a sharp increase in $R_{2}$ due to a small increase in the liposome membrane permeability. The simulation results imply that real-time, in-situ MRI measurements with AMF heating could provide high-resolution, high-contrast image of the liposomal site during the heating process.

In conclusion, this work provides a proof of concept for monitoring cargo (cancer drugs) release with dosage control and liposomal site simultaneously by MRI technique. We have used clinically approved materials such as liposome, SPIO, and Gd(III)-DTPA in our experiments to accelerate the chance of getting readily accepted for pre-clinical or translational MR theranostics. Future work should be done in vivo with real drug molecules, with active targeting by magnetofection, and with real-time, in-situ MRI measurements and AMF heating.

\section{Abbreviations}

AMF: alternating magnetic field; BBB: blood brain barrier; cryoTEM: transmission electron cryomicroscopy; DLS: dynamic light scattering; $\mathrm{D}_{\mathrm{pp}}$ : inter SPIO nanoparticle distance; EPR: enhanced permeability and retention; Fe: iron; Gd: Gadolinium; Gd(III)-DTPA: diethylenetriaminepentaacetic acid gadolinium(III); HIFU: high intensity focused ultrasound; ICP-OES: inductively coupled plasma optical emission spectrometry; MRI: magnetic resonance imaging; NMR: nuclear magnetic 
resonance; PBS: phosphate buffered saline; PEG: polyethelyne glycol; $\mathrm{R}_{1}$ : longitudinal relaxation rate; $\mathrm{r}_{1}$ : longitudinal relaxivity; $\mathrm{R}_{2}$ : transverse relaxation rate; $r_{p}$ : single SPIO radius; $r_{2}$ : transverse relaxivity; RES: reticuloendothelial system; RPT: Reciprocal Powered Time; SPIO: superparamagnetic iron oxide; $\tau_{\mathrm{CP}}$ : half of the interval between successive $180^{\circ}$ pulses in a CPMG sequence.

\section{Acknowledgements}

We gratefully acknowledge Mr. Ivo Atanasov, Dr. Peng Ge, Dr. Christian Beren for technical support and Prof. William Gelbart, Prof. Charles M. Knobler, Prof. Neil K. Garg, Prof. Andre Nel for lab facility. This work was supported by National Science Foundation (CHE-1112574, CHE-1416598), University of California Cancer Research Award (CRR-13-201412), and the Hirshberg Foundation for Pancreatic Cancer Research (YYL, ZL).

\section{Supplementary Material}

Supplementary figure.

http://www.ntno.org/v03p0166s1.pdf

\section{Competing Interests}

The authors have declared that no competing interest exists.

\section{References}

1. Kelkar SS, Reineke TM. Theranostics: combining imaging and therapy. Bioconjug. Chem. 2011; 22: 1879-1903

2. Wang $\mathrm{H}, \mathrm{Li} X, \mathrm{Tse} B W-\mathrm{C}$ et al. Indocyanine green-incorporating nanoparticles for cancer theranostics. Theranostics 2018; 8: 1227-1242

3. Janib SM, Moses AS, MacKay JA. Imaging and drug delivery using theranostic nanoparticles. Adv. Drug Deliv. Rev. 2010; 62: 1052-1063

4. Wang J, Liu L, You Q et al. All-in-one theranostic nanoplatform based on hollow MoS $x$ for photothermally-maneuvered oxygen self-enriched photodynamic therapy. Theranostics 2018; 8: 955-971

5. Sunderland CJ, Steiert M, Talmadge JE et al. Targeted nanoparticles for detecting and treating cancer. Drug Dev. Res. 2006; 67: 70-93

6. Tan J, Yang N, Zhong L et al. A new theranostic system based on endoglin aptamer conjugated fluorescent silica nanoparticles. Theranostics 2017; 7: $4862-4876$

7. Singh R, Lillard JW. Nanoparticle-based targeted drug delivery. Exp. Mol. Pathol. 2009; 86: 215-223

8. Brannon-Peppas L, Blanchette JO. Nanoparticle and targeted systems for cancer therapy. Adv. Drug Deliv. Rev. 2012; 64: 206-212

9. Parekh G, Shi Y, Zheng J et al. Nano-carriers for targeted delivery and biomedical imaging enhancement. Ther. Deliv. 2018; 9: 451-468

10. Hekman MC, Rijpkema M, Muselaers $\mathrm{CH}$ et al. Tumor-targeted dual-modality imaging to improve intraoperative visualization of clear cell renal cell carcinoma: a first in man study. Theranostics 2018; 8: 2161-2170

11. Ashton JR, Castle KD, Qi Y et al. Dual-energy CT imaging of tumor liposome delivery after gold nanoparticle-augmented radiation therapy. Theranostics 2018; 8: 1782-1797

12. Kong $X$, Dong B, Song $X$ et al. Dual turn-on fluorescence signal-based controlled release system for real-time monitoring of drug release dynamics in living cells and tumor tissues. Theranostics 2018; 8: 800-811

13. Slowing II, Vivero-Escoto JL, Wu C-W et al. Mesoporous silica nanoparticles as controlled release drug delivery and gene transfection carriers. Adv. Drug Deliv. Rev. 2008; 60: 1278-1288

14. Kotb S, Detappe A, Lux F et al. Gadolinium-based nanoparticles and radiation therapy for multiple brain melanoma metastases: Proof of concept before phase I trial. Theranostics 2016; 6: 418-427

15. Yen SK, Padmanabhan P, Selvan ST. Multifunctional iron oxide nanoparticles for diagnostics, therapy and macromolecule delivery. Theranostics 2013; 3: 986-1003

16. Hosta-Rigau L, Schattling P, Teo BM et al. Recent progress of liposomes in nanomedicine. J. Mater. Chem. B 2014; 2: 6686-6691

17. Béalle G, Di Corato R, Kolosnjaj-Tabi J et al. Ultra magnetic liposomes for MR imaging, targeting, and hyperthermia. Langmuir 2012; 28: 11834-11842

18. Pradhan P, Giri J, Rieken F et al. Targeted temperature sensitive magnetic liposomes for thermo-chemotherapy. J. Control. Release 2010; 142: 108-121
19. Mikhaylov G, Mikac U, Magaeva AA et al. Ferri-liposomes as an MRI-visible drug-delivery system for targeting tumours and their microenvironment. Nat. Nanotechnol. 2011; 6: 594-602

20. Amstad E, Kohlbrecher J, Müller E et al. Triggered release from liposomes through magnetic actuation of iron oxide nanoparticle containing membranes. Nano Lett. 2011; 11: 1664-1670

21. Zhang L, Cui H. HAase-sensitive dual-targeting irinotecan liposomes enhance the therapeutic efficacy of lung cancer in animals. Nanotheranostics 2018; 2: 280-294

22. Knights-Mitchell SS, Romanowski M. Near-infrared activated release of doxorubicin from plasmon resonant liposomes. Nanotheranostics 2018; 2: 295-305

23. Koning GA, Eggermont AMM, Lindner LH et al. Hyperthermia and thermosensitive liposomes for improved delivery of chemotherapeutic drugs to solid tumors. Pharm. Res. 2010; 27: 1750-1754

24. Allen TM, Cullis PR. Liposomal drug delivery systems: from concept to clinical applications. Adv. Drug Deliv. Rev. 2013; 65: 36-48

25. Meunier F, Prentice HG, Ringden O. Liposomal amphotericin B (AmBisome): safety data from a phase II/III clinical trial. J. Antimicrob. Chemother. 1991; 28: 83-91

26. Morris PE, Papadakos P, Russell JA et al. A double-blind placebo-controlled study to evaluate the safety and efficacy of L-2-oxothiazolidine-4-carboxylic acid in the treatment of patients with acute respiratory distress syndrome. Crit. Care Med. 2008; 36: 782-788

27. Rahman A, Treat J, Roh JK et al. A phase I clinical trial and pharmacokinetic evaluation of liposome-encapsulated doxorubicin. J. Clin. Oncol. 1990; 8: 1093-1100

28. Delanian S, Baillet F, Huart J et al. Successful treatment of radiation-induced fibrosis using liposomal CuZn superoxide dismutase: clinical trial. Radiother. Oncol. 1994; 32: 12-20

29. Hortobagyi GN, Ueno NT, Xia W et al. Cationic liposome-mediated E1A gene transfer to human breast and ovarian cancer cells and its biologic effects: a phase I clinical trial. J. Clin. Oncol. 2001; 19: 3422-3433

30. Northfelt DW, Dezube BJ, Thommes JA et al. Pegylated-liposomal doxorubicin versus doxorubicin, bleomycin, and vincristine in the treatment of AIDS-related Kaposi's sarcoma: results of a randomized phase III clinical trial. J. Clin. Oncol. 1998; 16: 2445-2451

31. Alberts DS, Muggia FM, Carmichael J et al. Efficacy and safety of liposomal anthracyclines in Phase I/II clinical trials. Semin. Oncol. 2004; 31: 53-90

32. Field SB, Bleehen NM. Hyperthermia in the treatment of cancer. Cancer Treat. Rev. 1979; 6: 63-94

33. Wust P, Hildebrandt B, Sreenivasa G et al. Hyperthermia in combined treatment of cancer. Lancet Oncol. 2002; 3: 487-497

34. Tagami T, Foltz WD, Ernsting MJ et al. MRI monitoring of intratumoral drug delivery and prediction of the therapeutic effect with a multifunctional thermosensitive liposome. Biomaterials 2011; 32: 6570-6578

35. Wang $\mathrm{C}, \mathrm{Hsu} \mathrm{CH}, \mathrm{Li} \mathrm{Z}$ et al. Effective heating of magnetic nanoparticle aggregates for in vivo nano-theranostic hyperthermia. Int. J. Nanomedicine 2017; 12: 6273-6287

36. Clavel CM, Nowak-Sliwinska P, Pounescu E et al. In vivo evaluation of small-molecule thermoresponsive anticancer drugs potentiated by hyperthermia. Chem. Sci. 2015; 6: 2795-2801

37. Ponce AM, Vujaskovic Z, Yuan F et al. Hyperthermia mediated liposomal drug delivery. Int. J. Hyperth. 2006; 22: 205-213

38. Unezaki S, Maruyama $\mathrm{K}$, Takahashi $\mathrm{N}$ et al. Enhanced delivery and antitumor activity of doxorubicin using long-circulating thermosensitive liposomes containing amphipathic polyethylene glycol in combination with local hyperthermia. Pharm. Res. 1994; 11: 1180-1185

39. Needham D, Anyarambhatla G, Kong G et al. A new temperature-sensitive liposome for use with mild hyperthermia: characterization and testing in a human tumor xenograft model. Cancer Res. 2000; 60: 1197-1201

40. Kong G, Braun RD, Dewhirst MW et al. Hyperthermia enables tumor-specific nanoparticle delivery: effect of particle size. Cancer Res. 2000; 60: 4440-4445

41. Lin FC, Hsu CH, Lin YY. Nano-therapeutic cancer immunotherapy using hyperthermia-induced heat shock proteins: insights from mathematical modeling. Int. J. Nanomedicine 2018; 13: 1-11

42. Boissenot $\mathrm{T}$, Bordat $\mathrm{A}$, Fattal $\mathrm{E}$ et al. Ultrasound-triggered drug delivery for cancer treatment using drug delivery systems: from theoretical considerations to practical applications. J. Control. Release 2016; 241: 144-163

43. Ranjan A, Jacobs GC, Woods DL et al. Image-guided drug delivery with magnetic resonance guided high intensity focused ultrasound and temperature sensitive liposomes in a rabbit Vx2 tumor model. J. Control. Release 2012; 158: 487-494

44. Lorenzato $\mathrm{C}$, Oerlemans $\mathrm{C}$, van Elk $\mathrm{M}$ et al. MRI monitoring of nanocarrier accumulation and release using Gadolinium-SPIO co-labelled thermosensitive liposomes. Contrast Media Mol. Imaging 2016; 11: 184-194

45. Tai LA, Tsai PJ, Wang YC et al. Thermosensitive liposomes entrapping iron oxide nanoparticles for controllable drug release. Nanotechnology 2009; 20: 135101

46. Thomas CR, Ferris DP, Lee J et al. Noninvasive remote controlled release of drug molecules in vitro using magnetic actuation of mechanised nanoparticles. J. Am. Chem. Soc. 2010; 132: 10623-10625

47. Saint-Cricq P, Deshayes S, Zink JI et al. Magnetic field activated drug delivery using thermodegradable azo-functionalised PEG-coated core-shell mesoporous silica nanoparticles. Nanoscale 2015; 7: 13168-13172

48. Rühle B, Datz S, Argyo C et al. A molecular nanocap activated by superparamagnetic heating for externally stimulated cargo release. Chem. Commun. 2016; 52: 1843-1846 
49. Banks WA, Gray AM, Erickson MA et al. Alternating magnetic field-induced hyperthermia increases iron oxide nanoparticle cell association/uptake and flux in blood- brain barrier models. Pharm. Res. 2015; 32: 1615-1625

50. Song X, Han X, Yu F et al. Polyamine-targeting gefitinib prodrug and its near-infrared fluorescent theranostic derivative for monitoring drug delivery and lung cancer therapy. Theranostics 2018; 8: 2217-2228

51. Li X, Schumann C, Albarqi HA et al. A tumor-activatable theranostic nanomedicine platform for NIR fluorescence-guided surgery and combinatorial phototherapy. Theranostics 2018; 8: 767-784

52. Langereis S, Hijnen N, Strijkers G et al. Research spotlight: multifunctional liposomes for MRI and image-guided drug delivery. Ther. Deliv. 2014; 5: 21-24

53. Ho LC, Hsu CH, Ou CM et al. Unibody core-shell smart polymer as a theranostic nanoparticle for drug delivery and MR imaging. Biomaterials 2015; 37: 436-446

54. Lorenzato C, Cernicanu A, Meyre ME et al. MRI contrast variation of thermosensitive magnetoliposomes triggered by focused ultrasound: A tool for image-guided local drug delivery. Contrast Media Mol. Imaging 2013; 8: 185-192

55. Ponce AM, Viglianti BL, Yu D et al. Magnetic Resonance imaging of temperature-sensitive liposome release: drug dose painting and antitumor effects. JNCI J. Natl. Cancer Inst. 2007; 99: 53-63

56. Talanov VS, Regino CAS, Kobayashi $\mathrm{H}$ et al. Dendrimer-based nanoprobe for dual modality magnetic resonance and fluorescence imaging. Nano Lett. 2006; 6: 1459-1463

57. Wen $\mathrm{S}, \mathrm{Li} \mathrm{K}$, Cai $\mathrm{H}$ et al. Multifunctional dendrimer-entrapped gold nanoparticles for dual mode CT/MR imaging applications. Biomaterials 2013; 34 : 1570-1580

58. Luo Y, Zhao L, Li X et al. The design of a multifunctional dendrimer-based nanoplatform for targeted dual mode SPECT/MR imaging of tumors. J. Mater. Chem. B 2016; 4: 7220-7225

59. Haribabu V, Farook AS, Goswami N et al. Optimized Mn-doped iron oxide nanoparticles entrapped in dendrimer for dual contrasting role in MRI. J. Biomed. Mater. Res. - Part B Appl. Biomater. 2016; 104: 817-824

60. Szpak A, Fiejdasz S, Prendota W et al. T1-T2 Dual-modal MRI contrast agents based on superparamagnetic iron oxide nanoparticles with surface attached gadolinium complexes. J. Nanoparticle Res. 2014; 16: 1-11

61. Zhou Z, Huang D, Bao J et al. A synergistically enhanced $T_{1}-T_{2}$ Dual-modal contrast agent. Adv. Mater. 2012; 24: 6223-6228

62. $\mathrm{Hu} \mathrm{F}$, Zhao YS. Inorganic nanoparticle-based $\mathrm{T} 1$ and $\mathrm{T} 1 / \mathrm{T} 2$ magnetic resonance contrast probes. Nanoscale 2012; 4: 6235-6243

63. Jung H, Park B, Lee $C$ et al. Dual MRI T1and T2(*)contrast with size-controlled iron oxide nanoparticles. Nanomedicine Nanotechnology, Biol. Med. 2014; 10: 1679-1689

64. Soenen SJH, Desender L, De Cuyper M. Complexation of gadolinium(III) ions on top of nanometre-sized magnetoliposomes. Int. J. Environ. Anal Chem. 2007; 87: 783-796

65. Shin T, Choi J, Yun S et al. T 1 and T 2 dual-mode MRI contrast agent for enhancing accuracy by engineered nanomaterials. ACS Nano 2014; 8: 3393-3401

66. Estelrich J, Sánchez-Martín MJ, Busquets MA. Nanoparticles in magnetic resonance imaging: From simple to dual contrast agents. Int. J. Nanomedicine 2015; 10: 1727-1741

67. De Cuyper M, Soenen SJH, Coenegrachts $\mathrm{K}$ et al. Surface functionalization of magnetoliposomes in view of improving iron oxide-based magnetic resonance imaging contrast agents: anchoring of gadolinium ions to a lipophilic chelate. Anal. Biochem. 2007; 367: 266-273

68. Chen J, Zhang WJ, Guo Z et al. PH-responsive iron manganese silicate nanoparticles as T1- T2* dual-modal imaging probes for tumor diagnosis. ACS Appl. Mater. Interfaces 2015; 7: 5373-5383

69. Huang HL, Lu PH, Yang HC et al. Fiber-optic triggered release of liposome in vivo: Implication of personalized chemotherapy. Int. J. Nanomedicine 2015; 10: 5171-5185

70. Paris JL, Cabañas MV, Manzano M et al. Polymer-grafted mesoporous silica nanoparticles as ultrasound-responsive drug carriers. ACS Nano 2015; 9: 11023-11033

71. Niesman MR, Khoobehi B, Peyman GA. Encapsulation of sodium fluorescein for dye release studies. Investig. Ophthalmol. Vis. Sci. 1992; 33: 2113-2119

72. Al-Jamal KT, Bai J, Wang JT-W et al. Magnetic drug targeting: preclinical in vivo studies, mathematical modeling, and extrapolation to humans. Nano Lett. 2016; 16: 5652-5660

73. T-w J, Bai J, T-W Wang J et al. Triple-modal imaging of magnetically-targeted nanocapsules in solid tumours in vivo. Theranostics 2016; 6: 342-356

74. Takeda K, Kobari M, Akaishi S et al. Targeting pancreatic chemotherapy cancer using liposome against antibody-combined. J. Exp. Med. 1994; 175: 29-42

75. D'Souza S. A review of in vitro drug release test methods for nano-sized dosage forms. Adv. Pharm. 2014; 2014: 1-12

76. Barzegar-Jalali M, Adibkia K, Valizadeh $\mathrm{H}$ et al. Kinetic analysis of drug release from nanoparticles. J. Pharm. Pharm. Sci. 2008; 11: 167-177

77. Beren CE. Effect of secondary structure on the size, configurational statistics, and packaging of long-RNA by viral capsid protein. Abstract of dissertation 2017

78. Liang ZP, Lauterbur PC, Principles of magnetic resonance imaging: a signal processing perspective. IEEE Engineering in Medicine and Biology Society, New York, US: SPIE Optical Engineering Press; 2000
79. Nel A, Ruoslahti E, Meng H. New insights into "permeability" as in the enhanced permeability and retention effect of cancer nanotherapeutics. ACS Nano 2017; 11: 9567-9569

80. Fang J, Nakamura H, Maeda H. The EPR effect: Unique features of tumor blood vessels for drug delivery, factors involved, and limitations and augmentation of the effect. Adv. Drug Deliv. Rev. 2011; 63: 136-151

81. Merbach A, Helm L, Tóth E et al. The chemistry of contrast agents in medical magnetic resonance imaging. 2nd ed. Chichester, UK: Wiley; 2013

82. Dong J, Zink JI. Taking the temperature of the interiors of magnetically heated nanoparticles. ACS Nano 2014; 8: 5199-5207

83. Yang C, City T. (19) United States (12) Patent Application Publication (10) Pub. No.: US 2009 / 0004258 A1 I05. 2009; 1

84. Vetrone F, Naccache R, Zamarrón A et al. Temperature sensing using fluorescent nanothermometers. ACS Nano 2010; 4: 3254-3258

85. Ye $\mathrm{F}, \mathrm{Wu} \mathrm{C}$, Jin $\mathrm{Y}$ et al. Ratiometric temperature sensing with semiconducting polymer dots. J. Am. Chem. Soc. 2011; 133: 8146-8149

86. Freddi S, Sironi L, D'Antuono R et al. A molecular thermometer for nanoparticles for optical hyperthermia. Nano Lett. 2013; 13: 2004-2010

87. Polo-Corrales L, Rinaldi C. Monitoring iron oxide nanoparticle surface temperature in an alternating magnetic field using thermoresponsive fluorescent polymers. J. Appl. Phys. 2012; 111: 07B334

88. Eze MO. Phase Transitions in phospholipid bilayers: lateral phase separations play vital roles in biomembranes. Biochem. Educ. 1991; 19: 204-208

89. Forbes N, Pallaoro A, Reich NO et al. Rapid, Reversible release from thermosensitive liposomes triggered by near-infra-red light. Part Part Syst. Charact. 2014; 31: 1158-1167

90. Paula S, Volkov AG, Van Hoek AN et al. Permeation of protons, potassium ions, and small polar molecules through phospholipid bilayers as a function of membrane thickness. Biophys J. 1996; 70: 339-348

91. Huster D, Jin AJ, Arnold K et al. Water permeability of polyunsaturated lipid membranes measured by ${ }^{17} \mathrm{O}$ NMR. Biophys J. 1997; 73: 855-864

92. Issa B, Obaidat IM, Hejasee RH, Qadri S, Haik Y. NMR relaxation in systems with magnetic nanoparticles: a temperature study. J Magn Reson Imaging. 2014; 39: 648-655

93. Matteucci M, Anyarambhatla G, Rosner G et al. Hyperthermia increases accumulation of technetium-99m-labeled liposomes in feline sarcomas. Clin Cancer Res 2000; 6: 3748-3755

94. Park K, Lee GY, Kim YS et al. Heparin-deoxycholic acid chemical conjugate as an anticancer drug carrier and its antitumor activity. J. Control. Release 2006; 114: 300-306

95. Oleson JR, Dewhirst MW, Harrelson JM et al. Tumor temperature distributions predict hyperthermia effect. Int. J. Radiat. Oncol. Biol. Phys. 1989; 16: 559-570

96. Cho $\mathrm{CH}$, Sreenivasa G, Plotkin $\mathrm{M}$ et al. Tumour perfusion assessment during regional hyperthermia treatment: Comparison of temperature probe measurement with H215O-PET perfusion. Int. J. Hyperth. 2010; 26: 404-411

97. Sun $X$, Xing L, Clifton Ling $C$ et al. The effect of mild temperature hyperthermia on tumour hypoxia and blood perfusion: Relevance for radiotherapy, vascular targeting and imaging. Int. J. Hyperth. 2010; 26: $224-231$ 